\title{
Balanço hídrico atual e simulações para cenários climáticos futuros no Município de Taubaté, SP, Brasil \\ (doi:10.4136/ambi-agua.25)
}

\author{
Andréa Sanae Horikoshi; Gilberto Fisch \\ Programa de Pós-Graduação em Ciências Ambientais da Universidade de Taubaté \\ Departamento de Ciências Agrárias \\ E-mail: andreasanae@hotmail.com; gfisch@uol.com.br
}

\section{RESUMO}

O objetivo deste trabalho foi elaborar o balanço hídrico atual para o município de Taubaté e analisar a questão da disponibilidade hídrica futura. Utilizou-se o método de Thornthwaite-Mather (1955) no cálculo do balanço hídrico climatológico, dados observacionais de precipitação e temperatura do ar (séries de 1965-2005 e 1992-2005) e dados simulados de temperatura e precipitação gerados pelo modelo HadCM3 (2010 a 2099). De uma forma geral, os cenários climáticos futuros apontam um aumento na temperatura do ar entre $+0,5^{\circ} \mathrm{C}$ a $+2,7^{\circ} \mathrm{C}$, associado a um aumento da precipitação entre 80 e $150 \mathrm{~mm}$. Esses resultados provocarão um aumento do déficit de água (ao redor de 50 a $80 \mathrm{~mm}$ ) e uma diminuição do excedente hídrico (próximo a 200mm). Esse aumento de precipitação não será suficiente para manter os níveis de evapotranspiração, pois este será muito maior, em função do aumento da temperatura do ar.

Palavras-chave: precipitação; evapotranspiração; modelo climático.

\section{Actual and future climatological budget for Taubaté, SP, Brazil}

\section{ABSTRACT}

The goal of this study was to determine the actual water budget for Taubaté, SP and investigate the future water availability. The Thornthwaite-Mather (1955) method was used for the climatological water budget combined with observed and simulated data of air temperature and precipitation. The future scenarios indicate an increase of temperature (ranging from $+0.5^{\circ} \mathrm{C}$ to $+2.7^{\circ} \mathrm{C}$ ) and precipitation (ranging from 80 to $150 \mathrm{~mm}$ ). These results will provoke an increase of the water deficit (ranging from 50 to $80 \mathrm{~mm}$ ) and a decrease of the surplus of water (around $200 \mathrm{~mm}$ ). The increase of the precipitation will not be sufficient to compensate for the increase of the water demands from evapotranspiration.

Keywords: rainfall; evapotranspiration; climatic simulation.

\section{INTRODUÇÃO}

A água é um elemento vital para a vida dos seres humanos e a sua disponibilidade possibilita o desenvolvimento tecnológico e social dos países. De toda água do planeta Terra, aproximadamente $97,3 \%$ é água salgada e apenas 2,7\% é de água doce. Da água doce disponível, $77,2 \%$ se encontram em forma de gelo nas calotas polares, 22,4\% se trata de água subterrânea, $0,35 \%$ se encontra nos lagos e pântanos, $0,04 \%$ se encontra na atmosfera e apenas 0,01\% está nos rios (Romera e Silva, 2003). 
A disponibilidade da água depende tanto das condições físicas da superfície da terra, quanto também das condições e variabilidade do clima, e afeta diretamente a vida animal e vegetal, e um dos seus mais conhecidos efeitos é a escassez de água. Segundo a FAO (2005), um em cada cinco países em desenvolvimento estará sofrendo de escassez de água até o ano de 2030, comprometendo a produção mundial de alimentos. Como conseqüência, poderá haver conflitos sociais.

Devido à grande demanda atual por recursos hídricos, faz-se necessário o conhecimento do ciclo da água dentro de uma bacia hidrográfica, principalmente das variáveis de precipitação e evapotranspiração. De acordo com Camargo (1971), para saber se uma região apresenta deficiência ou excesso de água durante o ano, é indispensável comparar dois elementos opostos do balanço hídrico: a precipitação que fornece a umidade para o solo e a evapotranspiração que consome a umidade do solo. Segundo Pereira et al. (2002), a disponibilidade hídrica pode ser quantificada pelo balanço hídrico climatológico, no qual fica evidenciada a flutuação temporal de períodos com excedente e com deficiência, permitindo, dessa forma, o planejamento das atividades agrícolas e a quantificação de irrigação.

O balanço hídrico é um método contábil de estimativa da disponibilidade de água no solo e baseia-se na aplicação do Princípio de Conservação de Massa (Pereira et al., 2002) em uma área de solo vegetado, contabilizando as entradas e saídas de água do solo. O balanço hídrico de uma região tende a se manter sem grandes alterações naturais, mas com o aumento populacional e o uso indiscriminado da água, as ações antrópicas podem acabar interferindo no ciclo hidrológico, por meio das taxas de precipitação, de infiltração no solo, da evapotranspiração e do escoamento superficial e profundo.

Segundo Nobre e Assad (2005), a temperatura média global do planeta à superfície vem aumentando nos últimos 120 anos, já tendo atingido $+0,6$ a $0,7^{\circ} \mathrm{C}$. A maior parte desse aquecimento ocorreu nos últimos 50 anos, e a última década apresentou os três anos mais quentes dos últimos 1000 anos da história recente da Terra. Recentemente, o relatório do IPCC (2007) reforçou esses fatos, associando como sendo muito provável que esse aumento de temperatura do ar seja conseqüência de ações antrópicas. Ainda segundo Nobre e Assad (2005), o aumento de temperatura induz a uma maior evapotranspiração, reduzindo a quantidade de água no solo, mesmo que as chuvas não diminuam significativamente. Esse fato pode causar a substituição dos biomas existentes hoje, por outros mais adaptados a climas com menor disponibilidade hídrica para as plantas, tais como savanas substituindo florestas, caatinga substituindo savanas, semi-deserto substituindo caatinga. Sendo assim, são importantes estimativas futuras do ciclo hidrológico.

Collischonn (2001) afirma que os modelos de circulação geral representam o comportamento tridimensional da atmosfera em todo o globo e podem ser utilizados para analisar as mudanças climáticas causadas pelas interferências antrópicas, tais como o desmatamento e/ou o aumento da concentração de gases de efeito estufa na atmosfera. Neste último caso, o aumento da concentração de gases de efeito estufa na atmosfera provoca uma mudança do balanço de energia calculado pelo modelo, com o conseqüente impacto nos elementos climáticos, tais como temperatura do ar e precipitação.

Leite (2004) em seu trabalho sobre a disponibilidade hídrica das águas superficiais da bacia hidrográfica do Rio Una, município de Taubaté, SP, obteve três cenários de disponibilidade versus demanda, que permitiu avaliar o grau de comprometimento da bacia e, constatou que, apesar da bacia não apresentar problema na sua disponibilidade hídrica no atual momento, em um futuro não muito distante, poderá se tornar uma bacia crítica passível de gerenciamento especial.

De acordo com Marengo e Alves (2005), a influência humana na forma de gerenciamento dos recursos hídricos, geração de energia, lançamento de esgotos nos rios, 
irrigação e crescimento populacional, pode estar impactando de forma negativa as vazões do Rio Paraíba do Sul, pois as variações de chuvas não parecem estar associadas à tendência negativa das vazões durante as observações dos postos fluviométricos dos últimos 50 anos.

O objetivo deste trabalho é avaliar a disponibilidade de água atual para o município de Taubaté, baseado no balanço hídrico climatológico e realizar estimativas para cenários climáticos futuros (até o ano de 2099) baseado em dados de temperatura e precipitação gerados por uma simulação climática.

\section{MATERIAL E MÉTODOS}

Conforme os resultados das análises de Fisch (1995), as principais características climáticas de Taubaté, SP são: um verão extremamente chuvoso (chove quase que diariamente no verão), e um inverno seco. $\mathrm{O}$ ano hidrológico inicia-se em agosto e termina em julho do ano seguinte, com $1.335 \mathrm{~mm}$ de total anual de precipitação com $42 \%$ da precipitação ocorrendo nos meses de dezembro a fevereiro.

Os dados meteorológicos necessários para a realização do Balanço Hídrico são temperatura do ar (para estimativas de evapotranspiração) e precipitação. Os dados de precipitação no período de 1983 a 2005 e os dados de temperatura no período de 1992 a 2005 foram coletados no posto meteorológico da Universidade de Taubaté (UNITAU). Além disso, utilizou-se também a série histórica do Departamento de Águas e Energia Elétrica (DAEE), posto prefixo E2-022, com um período de dados mais longo (1964-2005).

Para os cenários futuros foram utilizados dados mensais de precipitação e temperatura do ar entre os anos de 1950 e 2099, calculados pelo modelo HadCM3. Este algoritmo foi desenvolvido pelo Serviço Meteorológico Britânico (United Kingdom Meteorological Office) e é um modelo acoplado oceano-atmosfera, possuindo as seguintes características: a componente atmosférica tem 19 níveis com uma resolução espacial de 2,5 x 3,75 graus de latitude/longitude, produzindo uma grade global de $96 \times 73$ células, que equivalem a uma resolução de $417 \mathrm{~km}$ x $278 \mathrm{~km}$ na linha do Equador, passando para $295 \mathrm{~km}$ x $278 \mathrm{~km}$ a $45^{\circ}$ de latitude. Por outro lado, a componente oceânica possui 20 níveis de profundidade e uma resolução horizontal de 1,25 km x 1,25 km (Johns et al., 1997). Os principais processos de transferência de energia (radiação, convecção, transporte por nuvens, turbulência, etc.) são incorporados neste modelo. O intervalo de tempo é de 30 minutos para a componente atmosférica e de 1 hora para a parte oceânica.

Segundo Marengo (2006), os cenários de emissão representam uma visão possível do desenvolvimento futuro de emissões de gases de efeito estufa e aerossóis, que têm efeito radiativo potencial. Esses cenários são baseados numa combinação coerente e consistente de elevações, tais como, demografia, desenvolvimento socioeconômico e mudança na tecnologia. Assim como suas interações; enquanto o aumento dos gases de efeito estufa tende a aquecer o planeta, os aerossóis têm efeito de esfriamento.

Os dados dos cenários futuros foram obtidos a partir de simulações do modelo HadCM3, para o ponto de grade mais próximo da cidade de Taubaté. Essas simulações foram realizadas para dois cenários, a saber: Cenário A2 - cenário que descreve um mundo futuro muito heterogêneo no qual a regionalização é dominante, com alto índice de crescimento populacional, e menos preocupação em relação ao desenvolvimento econômico rápido, nesse mundo existirá um fortalecimento de identidades culturais regionais, com ênfase em valores da família e tradições locais; Cenário B2 - cenário que descreve um mundo futuro no qual a mudança tecnológica é mais diversa. A ênfase está em soluções locais, sustentabilidade econômica, social e ambiental, e nas iniciativas comunitárias e inovação social em lugar de soluções globais. Esses cenários são os utilizados pelos relatórios de Impacto Climático do 
Painel Intergovernamental de Mudanças Climáticas (Intergovernmental Panel of Climate Change - IPCC).

Ainda de acordo com Marengo (2006), existem fontes de incertezas na modelagem de clima para se fazer projeções de clima futuro em nível global ou regional na atual geração de modelos usados pelo IPCC, que devem ser considerados, a saber:

- Incerteza nas emissões futuras de gases de efeito estufa e aerossóis e das atividades vulcânicas e solares que afetam o forçamento radiativo do sistema climático;

- Incerteza na inclusão de efeitos diretos do aumento na concentração de $\mathrm{CO}_{2}$ atmosférico nas plantas e do efeito do comportamento das plantas no clima futuro;

- Incertezas na sensibilidade do clima global e nos padrões regionais das projeções do clima futuro simulado pelos modelos, devido às diferentes formas em que cada modelo de circulação geral da atmosfera representa os processos físicos e os mecanismos do sistema climático.

Rossato et al. (2004) transformaram os resultados de armazenamento médio mensal de água no solo, obtidos durante o período de 1971-1990, em percentual de água no solo, pela função da capacidade de água disponível (CAD). Ainda segundo Rossato et al. (2004), o Vale do Paraíba apresenta percentual do armazenamento de água no solo de $90-100 \%$ de janeiro a abril (período chuvoso) e de 70-90\% para os meses de estiagem. De acordo com Pereira et al. (2002), pode-se adotar CAD entre 75 e $100 \mathrm{~mm}$ para culturas anuais e entre 100 a $125 \mathrm{~mm}$ para culturas perenes. $\mathrm{O}$ valor de CAD igual a $100 \mathrm{~mm}$ praticamente satisfaz a todos esses resultados e foi assumido na elaboração do $\mathrm{BH}$.

\section{RESULTADOS E DISCUSSÃO}

A precipitação é uma variável que apresenta uma grande variabilidade temporal, principalmente na escala de tempo interanual. Embora a série temporal de dados do posto E2022 do DAEE seja longa (de 1964 a 2005), essa série possuía vários meses sem registro, provavelmente devido à pane no equipamento ou férias do observador meteorológico. Para completar essa série, utilizou-se o método da ponderação regional (Tucci et al., 2000), usando os dados disponíveis de três postos das redondezas. Esses postos vizinhos devem estar na mesma região climatológica e após o preenchimento da série de dados, é importante analisar a consistência dos dados. Um método muito utilizado para essa consistência é o método de Dupla Massa (Tucci et al., 2000).

A série de dados do posto E2-022 (representando Taubaté) foi preenchida com dados dos postos vizinhos E2-023 (Bacia do Rio Piracanguá, em Taubaté), E2-048 (Bacia de Caçapava Velha, em Caçapava) e E2-095 (Bacia do Rio Una, em Taubaté). Todos esses postos estão em uma distância inferior a $30 \mathrm{~km}$ de distância. A quantidade de dados mensais preenchidos no posto de E2-022 foi inferior a 6\%. Os dados preenchidos e consistidos foram comparados com os dados do posto meteorológico da UNITAU, que é uma série de dados mais curta, porém sem falhas. A Figura 1 mostra a consistência dos dados.

Com a série histórica de precipitação do posto E2-022 para o município de Taubaté, para o período de 1964 a 2005 completa e consistida, foi realizada uma análise sobre a variabilidade interanual da chuva e que está apresentada na Figura 2. A inserção de uma linha de tendência para os dados de totais anuais de chuva não mostra uma dependência temporal, apresentando uma precipitação total anual média de 1345,6 mm. Folhes e Fisch (2006) realizaram uma análise estatística detalhada da série de precipitação de Taubaté e comprovaram que não existe tendência anual da chuva na região. 
Horikoshi, A. S.; Fisch, G. Balanço hídrico atual e simulações para cenários climáticos futuros no Município de Taubaté, SP, Brasil. Ambi-Agua, Taubaté, v. 2, n. 2, p. 33-46, 2007. (doi:10.4136/ambi-agua.25)

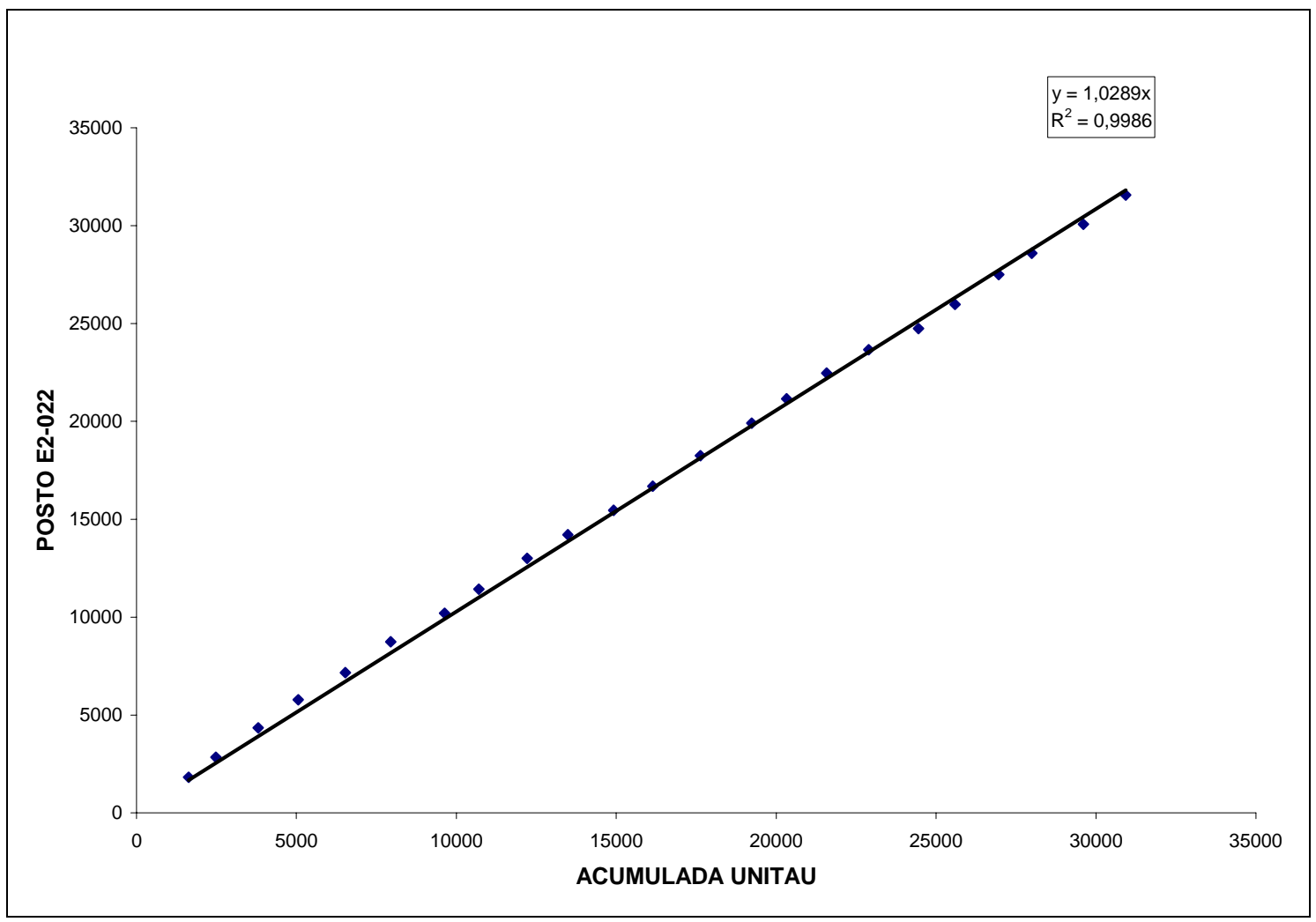

Figura 1. Relação entre os dados de precipitação acumulada do posto da UNITAU e o posto E222 do DAEE evidenciando a consistência espacial.

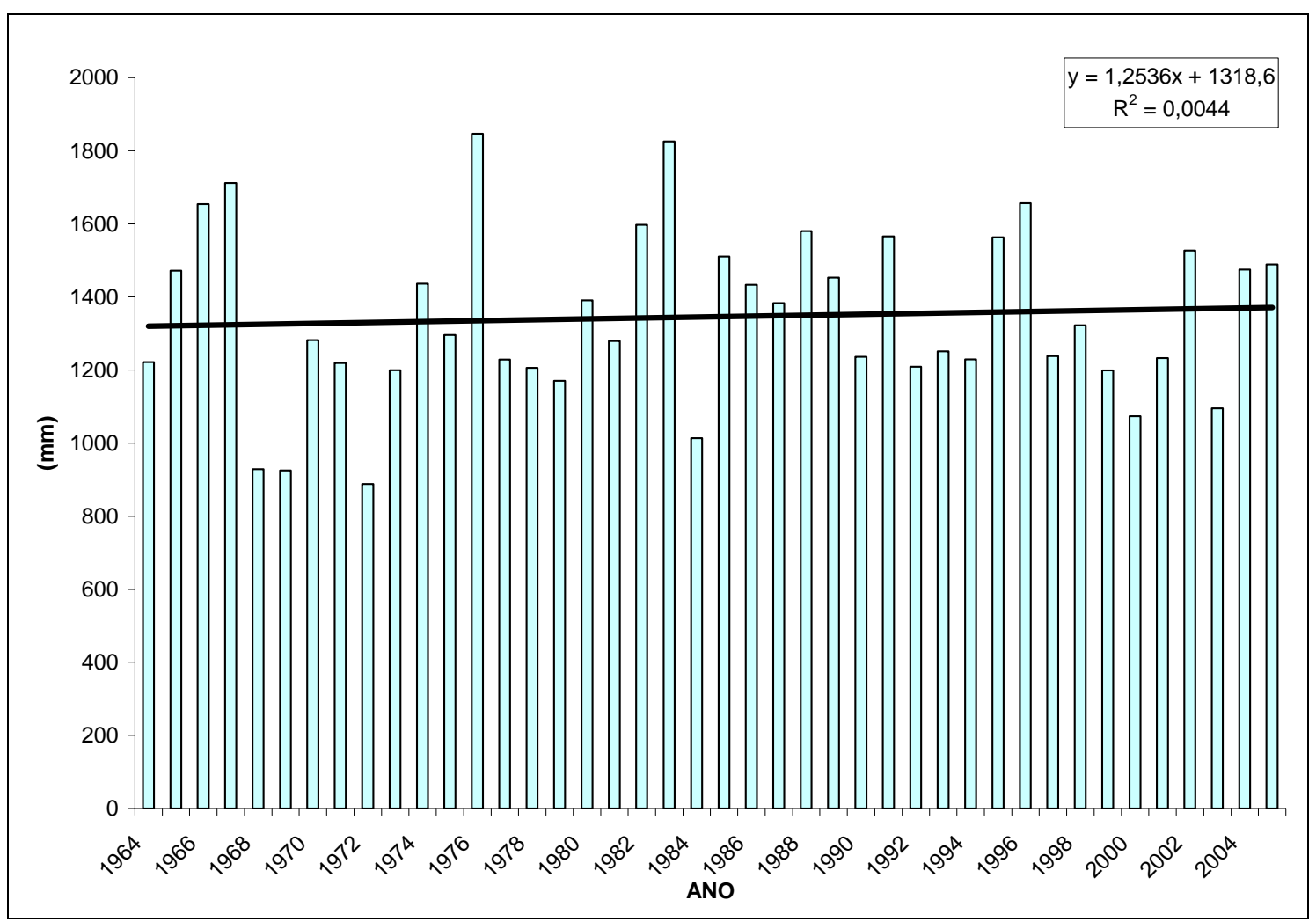

Figura 2. Precipitação total anual (mm) para o município de Taubaté, período 1964-2005. 

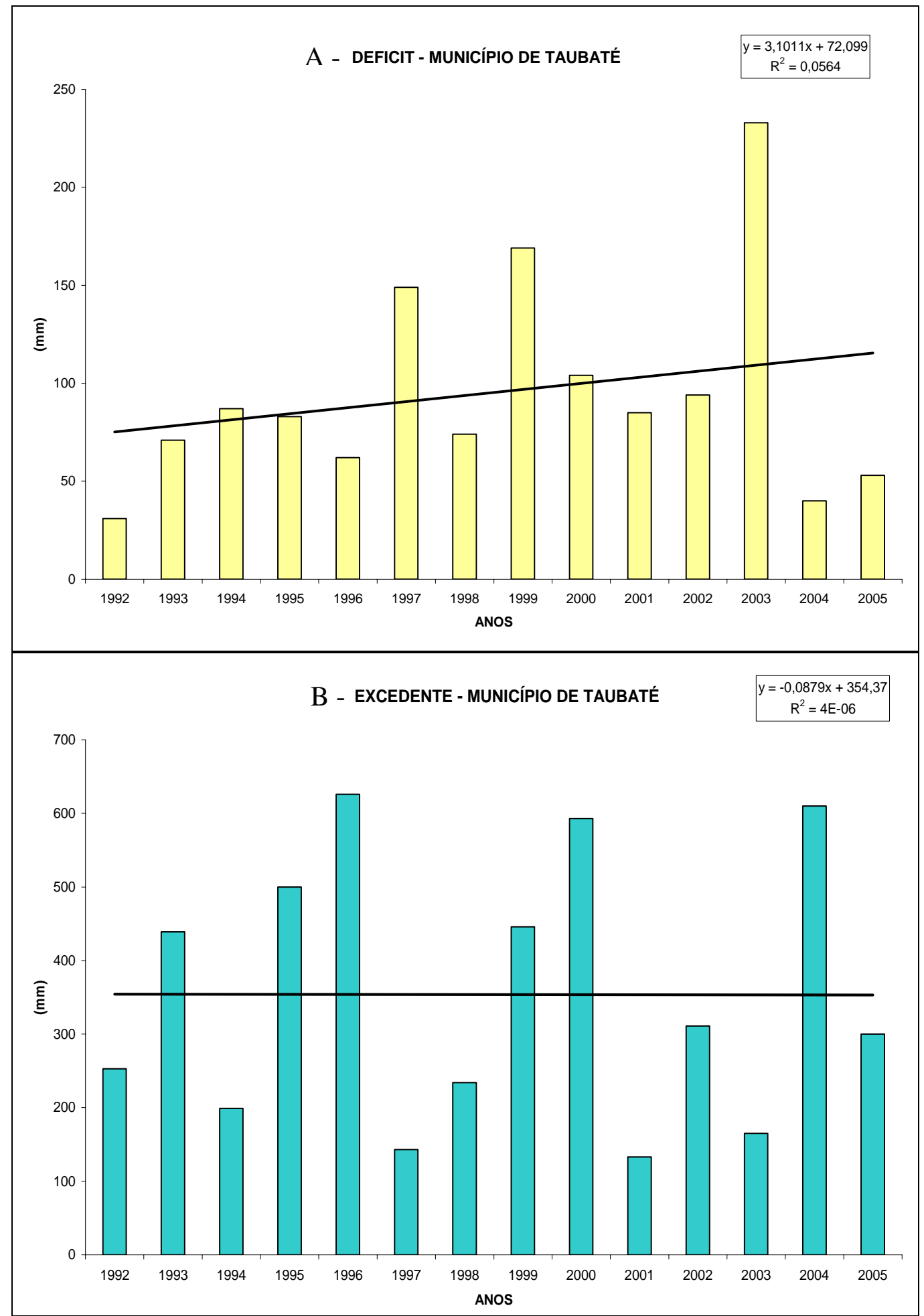

Figura 3. Série temporal (1992 a 2005) da Deficiência (A) e Excesso (B) de água para o Balanço Hídrico - I (Atual), em mm.

Na Figura 3, percebe-se uma tendência de aumento na quantidade de água no solo durante o período de estiagem, enquanto a linha de tendência do excedente hídrico parece estar estável. Entretanto, ressalta-se a alta variabilidade temporal, inerente dos dados de precipitação.

Segundo a metodologia de Thornthwaite e Mather (1955), a evapotranspiração é calculada em função da temperatura do ar. Como as previsões do modelo HadCM3 são em 
um ponto de grade que representa uma área grande (250 X $250 \mathrm{~km}^{2}$ aproximadamente) e a região de Taubaté possui um desnível topográfico acentuado (Serras do Mar e da Mantiqueira), optou-se por comparar e corrigir os dados de temperatura e precipitação do modelo HadCM3 em função dos dados observacionais do posto meteorológico da UNITAU, no período de 1992 a 2005. A comparação foi feita para o período de 1982-2005 para a precipitação e de 1992-2005 para a temperatura do ar. Os resultados da comparação do cenário B2 estão apresentados na Figura 4, sendo que os resultados para o cenário A2 são muito similares.

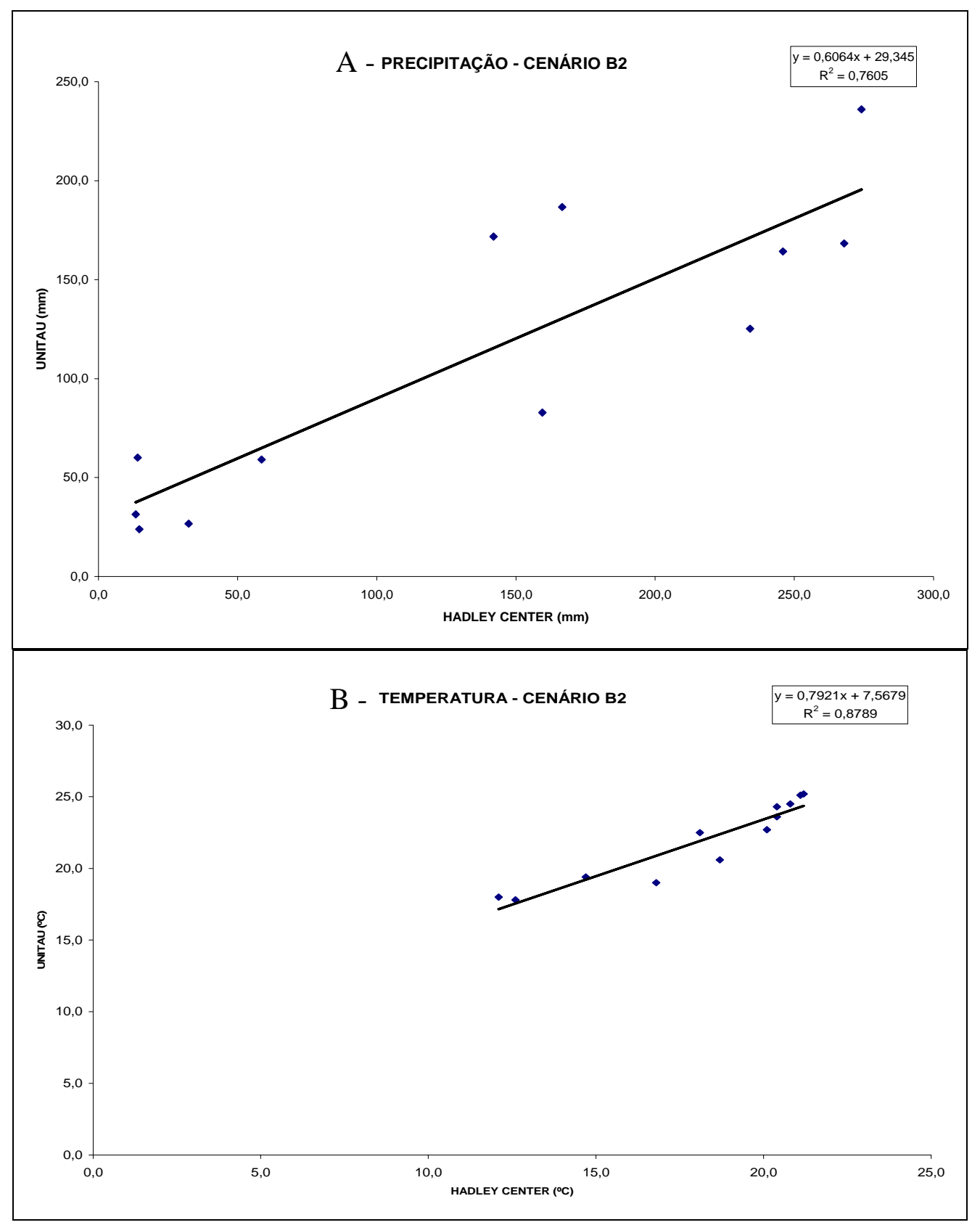

Figura 4. Relação entre os dados observacionais do posto da UNITAU e as simulações do HadCM3 para o elemento climático precipitação (A) e temperatura do $\operatorname{ar}(\mathrm{B})$.

Após essa análise observacional dos dados de precipitação e temperatura, realizou-se o Balanço Hídrico, para o período de 1992-2005 (considerado como sendo o atual regime 
hidrológico) e outros três períodos futuros: 2010-2029, 2040-2069 e 2070-2099. Todos esses três novos períodos tiveram uma média climatológica de trinta anos de dados, para observar as recomendações da Organização Mundial de Meteorologia (OMM). Esses cálculos estão apresentados nas Tabelas 1 (cenário atual), Tabelas 2, 4 e 6 para o cenário A2 e Tabelas 3, 5 e 7 para o cenário B2. Os valores de ETP calculados no BH-I (cenário atual) foram comparados com estimativas de evapotranspiração usando informações oriundas de tanque classe A (assumindo um coeficiente de tanque de 0,77), com um ótimo ajuste (coeficiente de correlação de 0,87 ). Isso garante a confiabilidade das estimativas.

Tabela 1. Balanço Hídrico - I: município de Taubaté (1992-2005).

\begin{tabular}{|c|c|c|c|c|c|c|c|c|c|c|c|}
\hline \multicolumn{12}{|c|}{ BALANÇOH'DRCOQMMTOLÓGCO- MUNĆPIODETAUBATÉ(1992-2005) } \\
\hline MÊS & $\mathbf{T}$ & $\begin{array}{l}\text { ETp } \\
\text { mm }\end{array}$ & Cor & $\begin{array}{l}\text { EIP } \\
\mathbf{~ m m}\end{array}$ & $\begin{array}{c}\mathbf{P} \\
\mathbf{m m}\end{array}$ & P-EIP & $\begin{array}{c}\text { ARM } \\
\mathbf{~ m m}\end{array}$ & $\begin{array}{l}\text { ALT } \\
\text { mm }\end{array}$ & $\begin{array}{l}\text { EIR } \\
\text { mm }\end{array}$ & $\begin{array}{l}\text { DEF } \\
\mathrm{mm}\end{array}$ & $\begin{array}{l}\text { EXC } \\
\mathrm{mm}\end{array}$ \\
\hline JAN & 25,0 & 116,75 & 1,15 & 134 & 236 & 102 & 100 & 0 & 134 & 0 & 102 \\
\hline \#V & 25,1 & 117,92 & 1,00 & 118 & 187 & 69 & 100 & 0 & 118 & 0 & 69 \\
\hline MAR & 24,2 & 107,67 & 1,05 & 113 & 172 & 59 & 100 & 0 & 113 & 0 & 59 \\
\hline ABR & 22,5 & 89,80 & 0,97 & 87 & 59 & -28 & 76 & -24 & 83 & 4 & 0 \\
\hline MA & 19,4 & 62,08 & 0,95 & 59 & 60 & 1 & 77 & 1 & 59 & 0 & 0 \\
\hline $\mathrm{JUN}$ & 18,0 & 51,52 & 0,89 & 46 & 24 & -22 & 61 & -15 & 39 & 7 & 0 \\
\hline JUL & 17,8 & 50,10 & 0,94 & 47 & 31 & -16 & 52 & -9 & 40 & 7 & 0 \\
\hline AGO & 18,9 & 58,17 & 0,98 & 57 & 27 & -30 & 39 & -14 & 41 & 16 & 0 \\
\hline SET & 20,6 & 72,09 & 1,00 & 72 & 83 & 11 & 50 & 11 & 72 & 0 & 0 \\
\hline का & 22,7 & 91,81 & 1,09 & 100 & 125 & 25 & 75 & 25 & 100 & 0 & 0 \\
\hline $\mathrm{NOV}$ & 23,5 & 100,08 & 1,10 & 110 & 164 & 54 & 100 & 25 & 110 & 0 & 29 \\
\hline DEZ & 24,5 & 111,02 & 1,17 & 130 & 168 & 38 & 100 & 0 & 130 & 0 & 38 \\
\hline ANO & 21,9 & -- & -- & 1073 & 1336 & 263 & - & 0 & 1039 & 34 & 297 \\
\hline
\end{tabular}

Tabela 2. Balanço Hídrico - II: Município de Taubaté (2010-2039) para o cenário A2.

\begin{tabular}{|c|c|c|c|c|c|c|c|c|c|c|c|}
\hline \multicolumn{12}{|c|}{ BALANÇO HÍDRICO CLMATOLÓGICO - MUNIĆ́PIO DE TAUBATÉ (2010-2039) } \\
\hline MÊS & $\begin{array}{c}\mathrm{T} \\
\left({ }^{\circ} \mathrm{C}\right)\end{array}$ & $\begin{array}{l}\text { ETp } \\
\text { mm }\end{array}$ & Cor & $\begin{array}{l}\text { EIP } \\
\text { mm }\end{array}$ & $\begin{array}{c}P \\
\mathbf{m m}\end{array}$ & P-ETP & $\begin{array}{l}\text { ARM } \\
\mathbf{m m}\end{array}$ & $\begin{array}{l}\text { ALT } \\
\text { mm }\end{array}$ & $\begin{array}{l}\text { EIR } \\
\text { mm }\end{array}$ & $\begin{array}{l}\text { DEF } \\
\mathrm{mm}\end{array}$ & $\begin{array}{l}\text { EXC } \\
\text { mm }\end{array}$ \\
\hline JAN & 25,1 & 117,36 & 1,15 & 135 & 178 & 43 & 100 & 0 & 135 & 0 & 43 \\
\hline FEV & 24,9 & 114,95 & 1,00 & 115 & 138 & 23 & 100 & 0 & 115 & 0 & 23 \\
\hline MAR & 24,1 & 105,60 & 1,05 & 111 & 111 & 0 & 100 & 0 & 111 & 0 & 0 \\
\hline ABR & 22,4 & 87,33 & 0,97 & 85 & 70 & $-15-$ & 86 & -14 & 84 & 1 & 0 \\
\hline MA & 19,5 & 60,91 & 0,95 & 58 & 49 & \begin{tabular}{l|l}
-9 \\
\end{tabular} & 79 & -7 & 56 & 2 & 0 \\
\hline JUN & 17,6 & 46,67 & 0,89 & 42 & 46 & 4 & 83 & 4 & 42 & 0 & 0 \\
\hline JUL & 18,2 & 50,92 & 0,94 & 48 & 46 & -2 & 81 & -2 & 48 & 0 & 0 \\
\hline AGO & 20,6 & 70,25 & 0,98 & 69 & 62 & -7 & 76 & -5 & 67 & 2 & 0 \\
\hline SET & 23,0 & 93,53 & 1,00 & 94 & 129 & 35 & 100 & 24 & 94 & 0 & 11 \\
\hline OUT & 24,1 & 105,60 & 1,09 & 115 & 192 & 77 & 100 & 0 & 115 & 0 & 77 \\
\hline NOV & 24,3 & 107,89 & 1,10 & 119 & 206 & 87 & 100 & 0 & 119 & 0 & 87 \\
\hline DEZ & 24,6 & 111,39 & 1,17 & 130 & 194 & 64 & 100 & 0 & 130 & 0 & 64 \\
\hline ANO & $\overline{\overline{22,4}}$ & " & -- & 1121 & 1421 & 300 & -- & $\overline{0}$ & 1116 & $\overline{5}$ & ב305 \\
\hline
\end{tabular}


Tabela 3. Balanço Hídrico - III: Município de Taubaté (2010-2039) para o cenário B2.

\begin{tabular}{|c|c|c|c|c|c|c|c|c|c|c|c|}
\hline \multicolumn{12}{|c|}{ BALANÇOHÍDRCOCMMATOLÓGCO- MUNáPIODETAUBATÉ(2010-2039) } \\
\hline MES & $\begin{array}{c}\mathrm{T} \\
\left({ }^{\circ} \mathrm{C}\right)\end{array}$ & $\begin{array}{l}\text { ETp } \\
\text { mm }\end{array}$ & Cor & $\begin{array}{l}\text { EIP } \\
\text { mm }\end{array}$ & $\begin{array}{c}\mathbf{P} \\
\mathbf{m m}\end{array}$ & P-ETP & $\begin{array}{c}\text { APM } \\
\text { mm }\end{array}$ & $\begin{array}{l}\text { ALT } \\
\text { mm }\end{array}$ & $\begin{array}{l}\text { EIR } \\
\mathrm{mm}\end{array}$ & $\begin{array}{l}\text { DEF } \\
\text { mm }\end{array}$ & $\begin{array}{l}\text { EXC } \\
\mathrm{mm}\end{array}$ \\
\hline JAN & 24,8 & 113,32 & 1,15 & 130 & 178 & 48 & 100 & 0 & 130 & 0 & 48 \\
\hline \#V & 24,8 & 113,32 & 1,00 & 113 & 148 & 35 & 100 & 0 & 113 & 0 & 35 \\
\hline MAR & 24,2 & 106,16 & 1,05 & 112 & 93 & $-19-$ & 83 & -17 & 110 & 2 & 0 \\
\hline ABR & 22,5 & 87,44 & 0,97 & 85 & 59 & -26 & 64 & -19 & 78 & 7 & 0 \\
\hline MA & 20,4 & 67,35 & 0,95 & 64 & 46 & -18 & 53 & -11 & 57 & 7 & 0 \\
\hline $\mathbf{J N}$ & 18,8 & 54,18 & 0,89 & 48 & 38 & -10 & 48 & -5 & 43 & 5 & 0 \\
\hline $\mathbf{T L}$ & 18,9 & 54,95 & 0,94 & 52 & 40 & -12 & 43 & -5 & 45 & 7 & 0 \\
\hline AGO & 21,5 & 77,47 & 0,98 & 76 & 48 & -28 & 32 & -10 & 58 & 18 & 0 \\
\hline SET & 23,3 & 95,97 & 1,00 & 96 & 106 & 10 & 42 & 10 & 96 & 0 & 0 \\
\hline OUT & 24,0 & 103,84 & 1,09 & 113 & 171 & 58 & 100 & 58 & 113 & 0 & 0 \\
\hline $\mathrm{NOV}$ & 24,3 & 107,33 & 1,10 & 118 & 180 & 62 & 100 & 0 & 118 & 0 & 62 \\
\hline DEZ & 24,8 & 113,32 & 1,17 & 133 & 198 & 65 & 100 & 0 & 133 & 0 & 65 \\
\hline ANO & $\overline{\overline{22,7}}$ & " & - & 1140 & 1305 & 165 & - & $\overline{0}$ & 1095 & 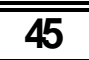 & 210 \\
\hline
\end{tabular}

Tabela 4. Balanço Hídrico - IV: Município de Taubaté (2040-2069) para o cenário A2.

\begin{tabular}{|c|c|c|c|c|c|c|c|c|c|c|c|}
\hline \multicolumn{12}{|c|}{ BALANÇOHÍDRCOCUMATOLÓGCO- MU NáPIODETALBATÉ(2040-2069) } \\
\hline MES & $\begin{array}{c}\mathrm{T} \\
{ }^{\circ} \mathrm{C}\end{array}$ & $\begin{array}{l}\text { ETp } \\
\text { mm }\end{array}$ & Cor & $\begin{array}{l}\text { EIP } \\
\text { mm }\end{array}$ & $\begin{array}{c}P \\
\mathbf{m m}\end{array}$ & P-ETP & $\begin{array}{l}\text { ARM } \\
\text { mm }\end{array}$ & $\begin{array}{l}\text { ALT } \\
\mathrm{mm}\end{array}$ & $\begin{array}{l}\text { EIR } \\
\text { mm }\end{array}$ & $\begin{array}{l}\mathrm{DE} \\
\mathrm{mm}\end{array}$ & $\begin{array}{l}\text { EXC } \\
\mathrm{mm}\end{array}$ \\
\hline JAN & 26,1 & 129,33 & 1,15 & 149 & 180 & 31 & 100 & 0 & 149 & 0 & 31 \\
\hline FV & 26,1 & 129,33 & 1,00 & 129 & 146 & 17 & 100 & 0 & 129 & 0 & 17 \\
\hline MAR & 25,3 & 117,98 & 1,05 & 124 & 111 & $-13-$ & 88 & -12 & 123 & 1 & 0 \\
\hline ABR & 23,8 & 98,50 & 0,97 & 96 & 67 & \begin{tabular}{|l|}
-29 \\
\end{tabular} & 66 & -22 & 89 & 7 & 0 \\
\hline MA & 21,4 & 71,97 & 0,95 & 68 & 49 & -19 & 54 & -11 & 60 & 8 & 0 \\
\hline $\mathbf{J N}$ & 19,7 & 56,37 & 0,89 & 50 & 47 & -3 & 53 & -2 & 49 & 1 & 0 \\
\hline $\mathbf{J L}$ & 19,9 & 58,08 & 0,94 & 55 & 46 & -9 & 48 & -5 & 51 & 4 & 0 \\
\hline AGO & 22,6 & 84,55 & 0,98 & 83 & 62 & -21 & 39 & -9 & 71 & 12 & 0 \\
\hline SET & 25,0 & 113,89 & 1,00 & 114 & 102 & -12 & 35 & -4 & 106 & 8 & 0 \\
\hline का & 25,6 & 122,15 & 1,09 & 133 & 191 & 58 & 93 & 58 & 133 & 0 & 0 \\
\hline NOV & 25,5 & 120,75 & 1,10 & 133 & 210 & 77 & 100 & 7 & 133 & 0 & 70 \\
\hline DEZ & 25,7 & 123,57 & 1,17 & 145 & 201 & 56 & 100 & 0 & 145 & 0 & 56 \\
\hline ANO & 23,9 & - & - & 1279 & 1412 & 133 & - & 0 & 1238 & 41 & 174 \\
\hline
\end{tabular}


Tabela 5. Balanço Hídrico - V: Município de Taubaté (2040-2069) para o cenário B2.

\begin{tabular}{|c|c|c|c|c|c|c|c|c|c|c|c|}
\hline \multicolumn{12}{|c|}{ BALANÇOHÍDRCOCLMATOLÓGCO- MUNáPIODETAUBATÉ(2040-2069) } \\
\hline MES & $\begin{array}{c}\mathrm{T} \\
\left({ }^{\circ} \mathrm{C}\right)\end{array}$ & $\begin{array}{l}\text { ETp } \\
\text { mm }\end{array}$ & Cor & $\begin{array}{l}\text { EIP } \\
\mathrm{mm}\end{array}$ & $\begin{array}{c}P \\
\mathbf{m m}\end{array}$ & P-ETP & $\begin{array}{c}\text { APM } \\
\text { mm }\end{array}$ & $\begin{array}{l}\text { ALT } \\
\text { mm }\end{array}$ & $\begin{array}{c}\text { EIR } \\
\mathrm{mm}\end{array}$ & $\begin{array}{l}\text { DEF } \\
\text { mm }\end{array}$ & $\begin{array}{l}\text { EXC } \\
\text { mm }\end{array}$ \\
\hline JAN & 25,5 & 121,19 & 1,15 & 139 & 146 & 7 & 100 & 0 & 139 & 0 & 7 \\
\hline 凡V & 25,5 & 121,19 & 1,00 & 121 & 133 & 12 & 100 & 0 & 121 & 0 & 12 \\
\hline MAR & 24,8 & 111,91 & 1,05 & 118 & 118 & 0 & 100 & 0 & 118 & 0 & 0 \\
\hline ABR & 23,4 & 94,76 & 0,97 & 92 & 58 & $-34-$ & 71 & -29 & 87 & 5 & 0 \\
\hline MA & 21,1 & 70,47 & 0,95 & 67 & 46 & -21 & 58 & -13 & 59 & 8 & 0 \\
\hline $\mathbf{J N}$ & 19,5 & 56,23 & 0,89 & 50 & 37 & -13 & 51 & -7 & 44 & 6 & 0 \\
\hline $\mathbf{J L}$ & 19,8 & 58,74 & 0,94 & 55 & 38 & -17 & 43 & -8 & 46 & 9 & 0 \\
\hline AGO & 22,5 & 84,70 & 0,98 & 83 & 49 & -34 & 30 & -12 & 61 & 22 & 0 \\
\hline SET & 25,1 & 115,83 & 1,00 & 116 & 93 & -23 & 24 & -6 & 99 & 17 & 0 \\
\hline OT & 25,2 & 117,16 & 1,09 & 128 & 173 & 45 & 69 & 45 & 128 & 0 & 0 \\
\hline $\mathrm{NOV}$ & 24,8 & 111,91 & 1,10 & 123 & 228 & 105 & 100 & 31 & 123 & 0 & 74 \\
\hline $\mathrm{DEZ}$ & 25,3 & 118,49 & 1,17 & 139 & 195 & 56 & 100 & 0 & 139 & 0 & 56 \\
\hline ANO & 23,5 & - & - & 1231 & 1314 & 83 & - & 0 & 1165 & 66 & 149 \\
\hline
\end{tabular}

Tabela 6. Balanço Hídrico - VI: Município de Taubaté (2070-2099) para o cenário A2.

\begin{tabular}{|c|c|c|c|c|c|c|c|c|c|c|c|}
\hline \multicolumn{12}{|c|}{ BALANÇOHÍDRCOCLMATOLÓGCO- MU NáPIODETALBATÉ(2070-2099) } \\
\hline MES & $\begin{array}{c}\mathrm{T} \\
\left({ }^{\circ} \mathrm{C}\right)\end{array}$ & $\begin{array}{l}\text { ETp } \\
\text { mm }\end{array}$ & Cor & $\begin{array}{l}\text { EIP } \\
\mathrm{mm}\end{array}$ & $\begin{array}{c}\mathbf{P} \\
\mathbf{m m}\end{array}$ & P-ETP & $\begin{array}{l}\text { ARM } \\
\text { mm }\end{array}$ & $\begin{array}{l}\text { ALT } \\
\text { mm }\end{array}$ & $\begin{array}{l}\text { EIR } \\
\text { mm }\end{array}$ & $\begin{array}{l}\text { DEF } \\
\mathrm{mm}\end{array}$ & $\begin{array}{l}\text { EXC } \\
\text { mm }\end{array}$ \\
\hline JAN & 27,4 & 144,70 & 1,15 & 166 & 191 & 25 & 100 & 0 & 166 & 0 & 25 \\
\hline \#V & 27,4 & 144,70 & 1,00 & 145 & 168 & 23 & 100 & 0 & 145 & 0 & 23 \\
\hline MAR & 26,6 & 137,48 & 1,05 & 144 & 120 & $-24-1$ & 79 & -21 & 141 & 3 & 0 \\
\hline ABR & 25,1 & 111,38 & 0,97 & 108 & 79 & \begin{tabular}{|l|}
-29 \\
\end{tabular} & 59 & -20 & 99 & 9 & 0 \\
\hline MA & 23,1 & 83,74 & 0,95 & 80 & 53 & -27 & 45 & -14 & 67 & 13 & 0 \\
\hline $\mathbf{J N}$ & 21,9 & 69,72 & 0,89 & 62 & 44 & -18 & 38 & -7 & 51 & 11 & 0 \\
\hline $\mathbf{J} \mathbf{L}$ & 22,2 & 73,06 & 0,94 & 69 & 51 & -18 & 31 & -6 & 57 & 12 & 0 \\
\hline AGO & 24,9 & 108,36 & 0,98 & 106 & 56 & -50 & 19 & -12 & 68 & 38 & 0 \\
\hline SET & 26,9 & 140,25 & 1,00 & 140 & 109 & -31 & 14 & -5 & 114 & 26 & 0 \\
\hline का & 27,3 & 143,83 & 1,09 & 157 & 180 & 23 & 37 & 23 & 157 & 0 & 0 \\
\hline $\mathrm{NOV}$ & 27,3 & 143,83 & 1,10 & 158 & 208 & 50 & 87 & 50 & 158 & 0 & 0 \\
\hline DEZ & 27,2 & 142,95 & 1,17 & 167 & 227 & 60 & 100 & 13 & 167 & 0 & 47 \\
\hline ANO & 25,6 & - & - & 1502 & 1486 & -16 & - & 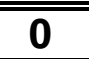 & 1391 & 1111 & 95 \\
\hline
\end{tabular}

Analisando-se o Balanço Hídrico atual (1992-2005), observa-se que há um total anual de precipitação de $1336 \mathrm{~mm}$, associado a uma evapotranspiração anual de $1073 \mathrm{~mm}$, com um superávit de água de $263 \mathrm{~mm}$. Esse Balanço Hídrico apresenta quatro meses de deficiência de água (total anual de $34 \mathrm{~mm}$ ) e cinco meses de excedente de água (totalizando $297 \mathrm{~mm}$ ). O 
armazenamento mínimo de água no solo foi de 39 mm. Resumindo, não ocorre falta de água, o aporte de água é superior a demanda evapotranspirativa.

A Tabela 8 apresenta um resumo das variações dos componentes do BH para os diferentes cenários, em relação aos elementos climáticos do Balanço Hídrico I, que representa as condições atuais.

Tabela 7. Balanço Hídrico - VII: Município de Taubaté (2070-2099) para o cenário B2.

\begin{tabular}{|c|c|c|c|c|c|c|c|c|c|c|c|}
\hline \multicolumn{12}{|c|}{ BALANÇOH'DRCOCMMATOLÓGCO- MUNáPIODETALBATÉ(2070-2099) } \\
\hline MES & $\begin{array}{c}\mathrm{T} \\
\left.{ }^{\circ} \mathrm{C}\right)\end{array}$ & $\begin{array}{l}\text { ETp } \\
\text { mm }\end{array}$ & Cor & $\begin{array}{l}\text { EIP } \\
\text { mm }\end{array}$ & $\begin{array}{c}P \\
\mathbf{m m}\end{array}$ & P-ETP & $\begin{array}{c}\text { ARM } \\
\text { mm }\end{array}$ & $\begin{array}{l}\text { ALT } \\
\text { mm }\end{array}$ & $\begin{array}{l}\text { EIR } \\
\mathbf{~ m m}\end{array}$ & $\begin{array}{l}\mathrm{DEF} \\
\mathrm{mm}\end{array}$ & $\begin{array}{l}\text { EXC } \\
\mathrm{mm}\end{array}$ \\
\hline JAN & 26,0 & 127,50 & 1,15 & 147 & 191 & 44 & 100 & 0 & 147 & 0 & 44 \\
\hline 円V & 26,1 & 129,00 & 1,00 & 129 & 162 & 33 & 100 & 0 & 129 & 0 & 33 \\
\hline MAR & 25,5 & 120,13 & 1,05 & 126 & 100 & $-26 \rightarrow$ & 77 & -23 & 123 & 3 & 0 \\
\hline ABR & 24,2 & 102,32 & 0,97 & 99 & 64 & -35 & 54 & -23 & 87 & 12 & 0 \\
\hline MA & 22,1 & 77,47 & 0,95 & 74 & 48 & -26 & 42 & -12 & 60 & 14 & 0 \\
\hline $\mathbf{J N}$ & 20,9 & 65,28 & 0,89 & 58 & 42 & -16 & 36 & -6 & 48 & 10 & 0 \\
\hline $\mathbf{U L}$ & 21,1 & 67,22 & 0,94 & ๘3 & 40 & -23 & 28 & -7 & 47 & 16 & 0 \\
\hline AGO & 23,3 & 91,10 & 0,98 & 89 & 51 & -38 & 19 & -9 & 60 & 29 & 0 \\
\hline SET & 25,1 & 114,44 & 1,00 & 114 & 108 & -6 & 18 & -1 & 109 & 5 & 0 \\
\hline का & 25,8 & 124,51 & 1,09 & 136 & 184 & 48 & 66 & 48 & 136 & 0 & 0 \\
\hline$\overline{\mathrm{NOV}}$ & 26,1 & 129,00 & 1,10 & 142 & 226 & 84 & 100 & 34 & 142 & 0 & 50 \\
\hline $\mathrm{DEZ}$ & 25,9 & 126,00 & 1,17 & 147 & 208 & 61 & 100 & 0 & 147 & 0 & 61 \\
\hline ANO & 24,3 & - & - & 1324 & 1424 & 100 & - & 0 & 1236 & 88 & 188 \\
\hline
\end{tabular}

Considerando-se o cenário A2 e os Balanços Hídricos para os períodos de 2010-2039 (BH-II), 2040-2069 (BH-IV) e 2070-2099 (BH-VI), observa-se que a precipitação aumenta durante esse período, bem como a evapotranspiração, uma vez que este último é função da temperatura do ar (que aumenta também), conforme é mostrado na Tabela 8. A diferença de água (precipitação - evapotranspiração) diminui abruptamente, principalmente para os últimos trinta anos, quando a evapotranspiração será superior a precipitação em $16 \mathrm{~mm}$. A deficiência de água chega a ser de até sete meses (para os casos de 2040-2069 e 2070-2099), com déficit integrados de $41 \mathrm{~mm}$ e $111 \mathrm{~mm}$, respectivamente. Associado a esse comportamento, o excedente diminui continuamente até atingir o valor de $95 \mathrm{~mm}$ no período de 2069-2070. Esse fato representa que a deficiência de água chega a atingir um valor de 77 mm a mais do que seu valor no BH-I (atual), associado a uma redução do excedente de 202 mm (Tabela 8).

Os números para o caso B2 também são similares, porém um pouco inferiores ao caso A2. Para o último período (2070-2099), a precipitação ainda é superior (100 mm) a evapotranspiração, o que provoca déficits de água menores e excedentes de água levemente superiores ao do cenário A2. Mas o comportamento geral é o mesmo. 
Tabela 8. Resumo das variações temporais dos componentes do Balanço Hídrico para os diferentes cenários A2 e B2, relativos ao Balanço Hídrico - I.

\begin{tabular}{l|c|c|c|c|c|c}
\hline \multirow{2}{*}{ Componentes do Balanço Hídrico } & \multicolumn{3}{c|}{ A2 } & \multicolumn{3}{c}{ B2 } \\
\cline { 2 - 7 } & \multicolumn{3}{|c}{} & \multicolumn{3}{c}{} \\
\cline { 2 - 8 } & BH-II & $\begin{array}{c}\text { BH- } \\
\text { IV }\end{array}$ & $\begin{array}{c}\text { BH- } \\
\text { VI }\end{array}$ & $\begin{array}{c}\text { BH- } \\
\text { III }\end{array}$ & BH-V & $\begin{array}{c}\text { BH- } \\
\text { VII }\end{array}$ \\
\hline Temperatura ( $\left.{ }^{\circ} \mathbf{C}\right)$ & $+0,5$ & $+2,0$ & $+3,7$ & $+0,8$ & $+1,6$ & $+2,4$ \\
Precipitação (mm) & +85 & +76 & +150 & -31 & -22 & +88 \\
Precipitação - Evapotranspiração (mm) & +300 & +133 & -16 & +165 & +83 & +100 \\
Deficiência (mm) & -29 & +7 & +77 & +11 & +32 & +54 \\
Excedente (mm) & +8 & -123 & -202 & -87 & -148 & -209 \\
\hline
\end{tabular}

\section{CONSIDERAÇÕES FINAIS}

De uma forma geral, os cenários climáticos futuros apontam um aumento na temperatura do ar, variando entre $+0,5^{\circ} \mathrm{C}$ a $+2,7^{\circ} \mathrm{C}$ para o cenário $\mathrm{A} 2$ e entre $+0,8^{\circ} \mathrm{C}$ e $+2,4^{\circ} \mathrm{C}$ para o cenário B2, aumento na precipitação (entre 85 e $150 \mathrm{~mm}$ para o cenário A2 e entre -31 e 88 mm para o cenário B2). Esses resultados provocarão um aumento do déficit de água (de até $77 \mathrm{~mm}$ para o período de 2070-2099 no cenário A2 e um pouco menor (54 mm) para o cenário B2), associado a uma diminuição do excedente hídrico (ao redor de $200 \mathrm{~mm}$ para ambos cenários). Em resumo, o clima estará mais quente, sendo que a estiagem será mais longa (de um a dois meses mais). O total anual da precipitação será um pouco mais elevado, com uma diminuição durante o verão e um leve aumento na época do inverno. Esse aumento de precipitação não será suficiente para manter os níveis de evapotranspiração, que será muito maior, em função do aumento da temperatura do ar. Esses resultados estão coerentes com as estimativas globais realizadas pelo Relatório Stern (Stern, 2006) e pelo IPCC (2007).

Recentemente, o CEIVAP (2006) publicou um relatório sobre o Plano de Recursos Hídricos na região do Rio Paraíba do Sul e apontou que, considerando o ano de 2005, não existe falta de água na Bacia para as atividades atuais. Esse fato também foi obtido pela análise do Balanço Hídrico - I e por Fisch (1995). Um ponto a ser considerado é que os estudos do CEIVAP (2006) somente levaram em conta as características hidroclimatológicas atuais (série temporal das vazões e precipitações), associadas a um crescimento urbano e demanda de água nas mesmas proporções. O ponto falho é não considerar os cenários climáticos futuros (como, por exemplo, nestas simulações do Hadley Center/CPTEC-INPE), pois a atmosfera tem um papel fundamental no ciclo hidrológico, por meio da ação do homem na liberação de gases do efeito estufa, balanço de radiação da atmosfera e também na formação de nuvens e chuvas correspondentes.

\section{AGRADECIMENTOS}

Ao Dr. José Antonio Marengo Orsini (CPTEC/INPE) pela cessão dos dados de simulação climática gerados pelo modelo HadCM3 e ao Departamento de Águas e Energia Elétrica - DAEE, na pessoa da Engenheira Marli Aparecida Maciel Leite. 


\section{REFERÊNCIAS}

CAMARgO, A. P. Balanço hídrico no Estado de São Paulo. Campinas: IAC, 1971. (Boletim Técnico, 116)

COLLISCHONN, W. Simulação hidrológica de grandes bacias. 2001. Tese (Doutorado em Engenharia) - Programa de Pós-Graduação em Engenharia de Recursos Hídricos e Saneamento Ambiental, Universidade Federal do Rio Grande do Sul, Porto Alegre, 2001.

COMITÊ DA BACIA DO RIO PARAÍBA DO SUL. Plano de recursos hídricos da Bacia do Rio Paraíba do Sul: resumo: diagnóstico dos recursos hídricos: relatório final. Resende: AGEVAP, 2006. Disponível em: <http://www.ceivap.org.br>. Acesso em: 21 fev. 2007.

FISCH, G. Caracterização climática e balanço hídrico de Taubaté (SP). Revista Biociências, Taubaté, v. 1, n. 1, p. 81-90, 1995.

FOLHES, M. T.; FISCH, G. Caracterização climática e estudo das tendências nas séries temporais de temperatura do ar e precipitação em Taubaté (SP). Ambiente e Água, Taubaté, v. 1, n. 1, p. 61-71, 2006.

FOOD AND AGRICULTURE ORGANIZATION. Crops and Drops. Roma: FAO, 2002. Disponível em: <http://www.fao.org/docrep>. Acesso em: 23 nov. 2005.

INTERGOVERNAMENTAL PANEL ON CLIMATE CHANGE. Climate change 2007: the physical science basis. Summary for policymakers. IPCC: Genebra, 2007. Disponível em: <http://www.ipcc.ch>. Acesso em: 02 fev. 2007.

JOHNS, T. C. et al. The second Hadley Centre coupled ocean-atmosphere GCM: model description, spinup and validation. Climate Dynamics, Berlin, v. 16, p. 103-134, 1997.

LEITE, M. A. R. M. Disponibilidade hídrica das águas superficiais da bacia hidrográfica do Rio Una. 2004. 65f. Monografia (Especialização em Gestão de Recursos Hídricos e Manejo de Bacias Hidrográficas) - Departamento de Ciências Agrárias, Universidade de Taubaté, Taubaté, 2004.

MARENGO, J. A. Mudanças climáticas globais e seus efeitos sobre a biodiversidade: caracterização do clima atual e definição das alterações climáticas para o território brasileiro ao longo do século XXI. Brasília: MMA: 2006.

MARENGO, J. A.; ALVES, L. M. Tendências hidrológicas da bacia do Rio Paraíba do Sul. Revista Brasileira de Meteorologia, Rio de Janeiro, v. 20, n. 2, p. 215-226, 2005.

NOBRE, C. A.; ASSAD, E. D. O aquecimento global e o impacto na Amazônia e na agricultura brasileira. INPE ePrint, São José dos Campos, v. 1, 2005. Disponível em: <http://eprint.sid.inpe.br/col/sid.inpe.br/ePrint@80/2005/09.12.12.51/doc/v1.pdf>. Acesso em: 23 março 2007.

PEREIRA, A. R.; ANGElOCCI, L. R.; SENTELHAS, P. C. Agrometeorologia: fundamentos e aplicações práticas. Guaíba: Agropecuária, 2002. 478p.

ROMERA E SILVA, P. A. (org). Água: quem vive sem? 2. ed. São Paulo: FCTH / CT-Hidro, 2003. 
ROSSATO, L.; ALVALÁ R. C. S.; TOMASELLA, J. Variação espaço-temporal da umidade do solo no Brasil: análise das condições médias para o período de 1971-1990. Revista Brasileira de Meteorologia, Rio de Janeiro, v.19, n. 2, p. 113-122, 2004.

STERN, N. Stern review on the economics of climate change. 2006. Disponível em: <http://www.hm-treasury.gov.uk>. Acesso em: 8 dez. 2006.

THORNTHWAITE, C. W.; MATHER, R. J. The water balance. New Jersey: Laboratory of climatology, 1955. 104 p. (Publication in Climatology, 8).

TUCCI, C. E. M. et al. Hidrologia: ciência e aplicação. 2. ed. Porto Alegre: UFRS, 2000. 943p. 